\title{
Salicylic Acid Regulates Basal Resistance to Fusarium Head Blight in Wheat
}

\author{
Ragiba Makandar, ${ }^{1,2}$ Vamsi J. Nalam, ${ }^{1}$ Hyeonju Lee, ${ }^{3}$ Harold N. Trick, ${ }^{3}$ Yanhong Dong, ${ }^{4}$ and Jyoti Shah ${ }^{1}$ \\ ${ }^{1}$ Department of Biological Sciences, University of North Texas, Denton 76203, U.S.A.; ${ }^{2}$ Department of Plant Sciences, \\ University of Hyderabad, Gachibowli, Hyderabad, India; ${ }^{3}$ Department of Plant Pathology, Kansas State University, \\ Manhattan 66506, U.S.A.; ${ }^{4}$ Department of Plant Pathology, University Minnesota, St. Paul 55108, U.S.A.
}

Submitted 8 September 2011. Accepted 14 November 2011.

\begin{abstract}
Fusarium head blight (FHB) is a destructive disease of cereal crops such as wheat and barley. Previously, expression in wheat of the Arabidopsis NPR1 gene (AtNPR1), which encodes a key regulator of salicylic acid (SA) signaling, was shown to reduce severity of FHB caused by Fusarium graminearum. It was hypothesized that $\mathrm{SA}$ signaling contributes to wheat defense against $F$. graminearum. Here, we show that increased accumulation of $\mathrm{SA}$ in fungusinfected spikes correlated with elevated expression of the SA-inducible pathogenesis-related 1 (PRI) gene and FHB resistance. In addition, FHB severity and mycotoxin accumulation were curtailed in wheat plants treated with SA and in AtNPR1 wheat, which is hyper-responsive to SA. In support of a critical role for SA in basal resistance to FHB, disease severity was higher in wheat expressing the $N a h G$ encoded salicylate hydroxylase, which metabolizes $\mathrm{SA}$. The FHB-promoting effect of $N a h G$ was overcome by application of benzo $(1,2,3)$, thiadiazole-7 carbothioic acid $S$-methyl ester, a synthetic functional analog of $\mathrm{SA}$, thus confirming an important role for $\mathrm{SA}$ signaling in basal resistance to FHB. We further demonstrate that jasmonate signaling has a dichotomous role in wheat interaction with $F$. graminearum, constraining activation of SA signaling during early stages of infection and promoting resistance during the later stages of infection.
\end{abstract}

Plants are exposed to a wide variety of pathogens in nature and have evolved complex strategies to counter these threats. Phytohormones such as jasmonic acid (JA) and salicylic acid (SA) contribute to plant defense (Browse 2009; Chaturvedi and Shah 2007; Durrant and Dong 2004). Whereas the JA-regulated mechanisms primarily contribute to defense against necrotrophic pathogens that derive nutrients from dead host cells, SA primarily contributes to pathogens that display a biotrophic phase in their life cycle (Glazebrook 2005). The role of these two mechanisms in plant defense has been well characterized in the dicot plant Arabidopsis thaliana (Balbi and Devoto 2008; Chaturvedi and Shah 2007; Durrant and Dong 2004; Wasternack 2007). For example, JA signaling contributes to Arabidopsis defense against the fungal pathogens Botrytis cinerea and Alternaria brassicicola, the oomycete Pythium irregulare, and the bacterial

R. Makandar and V. J. Nalam contributed equally to this work.

Corresponding author: J. Shah; E-mail: Shah@unt.edu;

Telephone: +1.940.565.3535; Fax: +1.940.565.4136;

* The $\boldsymbol{e}$-Xtra logo stands for "electronic extra" and indicates that four supplementary figures are published online. pathogen Erwinia carotovora (Ferrari et al. 2003; NormanSetterblad et al. 2000; Staswick and Tiryaki 2004; Staswick et al. 1998; Thomma et al. 2000), while SA-regulated mechanisms curtail infection caused by the oomycete Hyaloperonospora arabidopsidis, the bacterial pathogen Pseudomonas syringae and the fungal pathogen Erysiphe orontii (Cao et al. 1994; Delaney et al. 1995; Reuber et al. 1998; Shah et al. 1997). JA and SA are also involved in the systemic activation of defenses. JA is required for the activation of induced systemic resistance (ISR), which is expressed in the foliage in response to colonization of roots by some rhizobacterial species (Verhagen et al. 2004), and SA is required for systemic acquired resistance (SAR), which is activated in the uninfected leaves in response to a localized infection of another leaf (Chaturvedi and Shah 2007; Durrant and Dong 2004; Métraux et al. 2002). Cross-talk between SA and JA signaling further fine tunes defenses (Pieterse et al. 2009). For example, SA can antagonize the activation of JA-dependent defenses in Arabidopsis leaves (Spoel et al. 2007). Similarly, promotion of SA accumulation and signaling in response to pathogen infection can be marginalized by JA (Kloek et al. 2001; Spoel et al. 2007). Despite the well-documented antagonism between SA and JA signaling, both SA and JA are required for the full extent of basal resistance against Pythium irregulare, Pseudomonas syringae, and B. cinerea (Adie et al. 2007; Berrocal-Lobo et al. 2002; Clarke et al. 2000; Ferrari et al. 2003; Thomma et al. 1998, 1999; Zimmerli et al. 2001), thus highlighting the complexity in the involvement of SA and JA in plant defense.

Several fungi in the genus Fusarium are important pathogens of plants. A complex of closely related Fusarium spp. is responsible for Fusarium head blight (FHB) disease of wheat and other small grains (Xu and Nicholson 2009). Fusarium graminearum Schwabe (Gibberella zeae (Schwein.) Petch) is the major causative agent of FHB in the wheat-growing regions around the world (Bai and Shaner 2004; Xu and Nicholson 2009). F. graminearum infection of wheat florets is initiated at anthesis. The fungus subsequently spreads to other spikelets, eventually resulting in necrosis and bleaching of the spikelets (McMullen et al. 1997a). Stomates are potential entry points for the fungus, which can also directly penetrate the host surface. Infection is likely facilitated by a variety of hydrolases produced by the fungus (Walter et al. 2010). Although $F$. graminearum is a necrotrophic phytopathogen, during the early stages of infection a biotrophic phase has been described when fungal penetration of host cells is not observed and fungal growth is limited to extracellular spaces (Brown et al. 2010; Goswami and Kistler 2004). In addition to limiting grain yield, the fungus produces toxins such as deoxynivalenol (DON) that render grain from infected plants unsuitable for 
human and animal consumption (Bai and Shaner 2004; McMullen et al. 1997b). In the absence of a monogenic source of resistance against FHB, quantitative trait loci (QTL) from various sources have been introgressed to develop wheat varieties with enhanced FHB resistance (Buerstmayr et al. 2009). However, the protection provided by these QTL is insufficient during FHB epidemics.

Despite the economic importance of FHB to agriculture, current understanding of signaling mechanisms that regulate plant defense against $F$. graminearum is limited. Recent studies suggest that JA signaling has a role in controlling FHB infestation in wheat. Expression of wheat genes that are homologous to JA-inducible genes from other plant species, as well as those involved in JA synthesis, were upregulated in $F$. graminearum-infected spikes (Ding et al. 2011; Li and Yen 2008). Furthermore, FHB resistance was enhanced when wheat spikes were repeatedly sprayed with methyl-JA (MeJA), thus leading to the suggestion that JA has an important contribution to wheat defense against $F$. graminearum ( $\mathrm{Li}$ and $\mathrm{Yen}$ 2008). Indeed, JA levels increase in F. graminearum-inoculated wheat spikes (Ding et al. 2011), thus supporting the notion that JA signaling has a role in plant interaction with $F$. graminearum. In Arabidopsis, which is also colonized by $F$. graminearum (Chen et al. 2006, 2009; Cuzick et al. 2008; Makandar et al. 2006, 2010; Savitch et al. 2007; Skadsen and Hohn 2004; Urban et al. 2002; Van Hemelrijck et al. 2006), JA has a dichotomous role in plant interaction with $F$. graminearum (Makandar et al. 2010). During the early stages of infection, JA signaling contributed to susceptibility to disease caused by $F$. graminearum. However, during the later stages of infection, JA promoted resistance.

F. graminearum-infected spikes also express elevated levels of a family of pathogenesis-related $(P R)$ genes (Ding et al. 2011; Pritsch et al. 2000; Yu and Muehlbauer 2001). In Arabidopsis and many other dicots, expression of related $P R$ genes is associated with the induction of SA signaling (Chaturvedi and Shah 2007; Durrant and Dong 2004). Molecular-genetic studies in Arabidopsis have confirmed an important role for $\mathrm{SA}$ in basal resistance to $F$. graminearum (Makandar et al. 2010; Savitch et al. 2007). Severity of disease caused by $F$. graminearum was elevated in the SA biosynthesis sid2 mutant of Arabidopsis and in transgenic Arabidopsis plants expressing the NahG gene, which encodes an SA-degrading salicylate hydroxylase that hydrolyzes SA to yield catechol (Makandar et al. 2010; Savitch et al. 2007). In addition, the SA signaling-deficient Arabidopsis nprl mutant exhibited enhanced severity of disease caused by $F$. graminearum and $F$. culmorum, another causal agent of FHB in small-grain cereals (Cuzick et al. 2008; Makandar et al. 2010). By contrast, constitutive overexpression of NPRl in Arabidopsis resulted in enhanced resistance against $F$. graminearum (Makandar et al. 2010). Resistance against $F$. graminearum was also enhanced in Arabidopsis plants in which SAR was induced by a prior inoculation with a bacterial pathogen or by a SAR-inducing activity purified from Arabidopsis (Makandar et al. 2010). Similarly, FHB resistance was enhanced in wheat spikes that were locally treated with a SAR-inducing activity purified from Arabidopsis (Chaturvedi et al. 2008). In addition, the constitutively elevated accumulation of SA in a transgenic wheat line was accompanied by heightened FHB resistance (Anand et al. 2003a). Furthermore, constitutive expression in transgenic wheat of the Arabidopsis NPRl (AtNPRl) transcript, was accompanied by enhanced basal resistance to $F$. graminearum (Makandar et al. 2006). AtNPRl-expressing wheat also exhibited enhanced sensitivity to benzo $(1,2,3)$, thiadiazole-7 carbothioic acid $S$-methyl ester (BTH), a synthetic functional analog of SA. Expression of the wheat $P R l$ gene was induced more rapidly and strongly in
BTH-treated AtNPRl-expressing transgenic wheat than in nontransgenic plants (Makandar et al. 2006). F. graminearuminfected spikes also accumulated elevated levels of SA (Ding et al. 2011), thus raising the possibility of SA's involvement in wheat defense against $F$. graminearum. However, other studies showed that SA and BTH sprayed onto wheat spikes were ineffective in enhancing FHB, leading to the suggestion that SA signaling does not contribute to FHB resistance in wheat (Li and Yen 2008; Yu and Muehlbauer 2001). Thus, whether wheat utilizes SA signaling for controlling FHB is unclear.

Here, we demonstrate that FHB resistance in AtNPRlexpressing wheat is accompanied by stronger promotion of SA accumulation and a SAR-like mechanism than in nontransgenic plants. In addition, SA applied as a soil drench effectively enhanced FHB resistance in wheat. The importance of SA in basal resistance against FHB was confirmed in experiments with transgenic NahG wheat, which expresses an SAdegrading salicylate hydroxylase. NahG wheat exhibited heightened severity to FHB which could be overcome by soil-drench application of BTH, thus supporting the suggestion that SA signaling has a critical role in basal resistance against FHB. We further demonstrate that MeJA, when applied during the early stages of infection, suppressed the FHB resistance-enhancing effect of AtNPR1. By contrast, during later stages of infection, MeJA enhanced resistance to FHB, indicating a complex interaction between SA/NPR1 and JA signaling in wheat interaction with $F$. graminearum.

\section{RESULTS}

\section{F. graminearum infection results in systemic $P R 1$ expression and accumulation of $\mathrm{SA}$ in wheat spikes, which is enhanced by AtNPR1 expression.}

In Arabidopsis and tobacco, SAR is accompanied by systemic increase in SA content and upregulation of $P R 1$ expression in the uninfected organs of a plant that was locally infected with a pathogen (Durrant and Dong 2004; Métraux et al. 2002). Previously, point inoculation of a few spikelets in wheat with $F$. graminearum was shown to result in systemic expression of $P R I$ in the uninoculated spikelets in the same spike (Pritsch et al. 2001). Similarly, we observed that $P R I$ was expressed at elevated levels in the $F$. graminearum-inoculated central and the lower uninoculated spikelet of the wheat 'Sumai-3', which was used as an FHB-resistant control (Fig. 1A). To determine whether the systemic induction of PRl expression is accompanied by an increase in SA, as seen in dicots, we monitored SA content in the $F$. graminearum-inoculated (central) spikelet and the uninoculated (lower) spikelet of Sumai-3. SA content was significantly higher in the central and the lower spikelets compared with the corresponding spikelets of mock-inoculated Sumai-3 (Fig. 1B), thus confirming that systemic enhancement of $P R 1$ expression in F. graminearum-infected spikes is accompanied by a systemic increase in SA content in the FHB-resistant Sumai-3. By contrast, a corresponding increase in SA was not observed in the $F$. graminearum-treated spikes of 'Bobwhite' wheat (Fig. 1B), which exhibits enhanced susceptibility to $F$. graminearum compared with Sumai-3 (Makandar et al. 2006). Furthermore, PRI expression in F. graminearum-inoculated (central) and lower uninoculated spikelets of Bobwhite was weaker than in Sumai-3 (Fig. 1A). Compared with nontransgenic Bobwhite, constitutive expression of AtNPRl in transgenic wheat was associated with a systemic increase in SA accumulation and $P R I$ expression in response to $F$. graminearum infection (Fig. 1A and B). Taken together, these data indicate that $F$. graminearum-infection results in the activation of a SAR-like response, which can be enhanced in the FHB-susceptible wheat Bobwhite by constitutive expression of AtNPRl. 
SA application curtails FHB infection in wheat.

Because AtNPRI expression promotes SA accumulation and $P R 1$ expression in $F$. graminearum-infected wheat, and AtNPRl is involved in SA signaling, we hypothesized that activation of SA signaling can control FHB severity in wheat. However, a previous study demonstrated that SA sprayed onto wheat spikes was ineffective in enhancing FHB resistance ( $\mathrm{Li}$ and Yen 2008). However, whether this treatment resulted in successful build up of SA in spikes was not determined. Therefore, we reevaluated the impact of SA on FHB using a soil-drench application, which resulted in systemic increase in SA content (Fig. 2A). SA content was twofold higher in spikes of Bobwhite plants treated with SA compared with control plants treated with water. Spikes of SAtreated Bobwhite plants also expressed PRI at higher levels
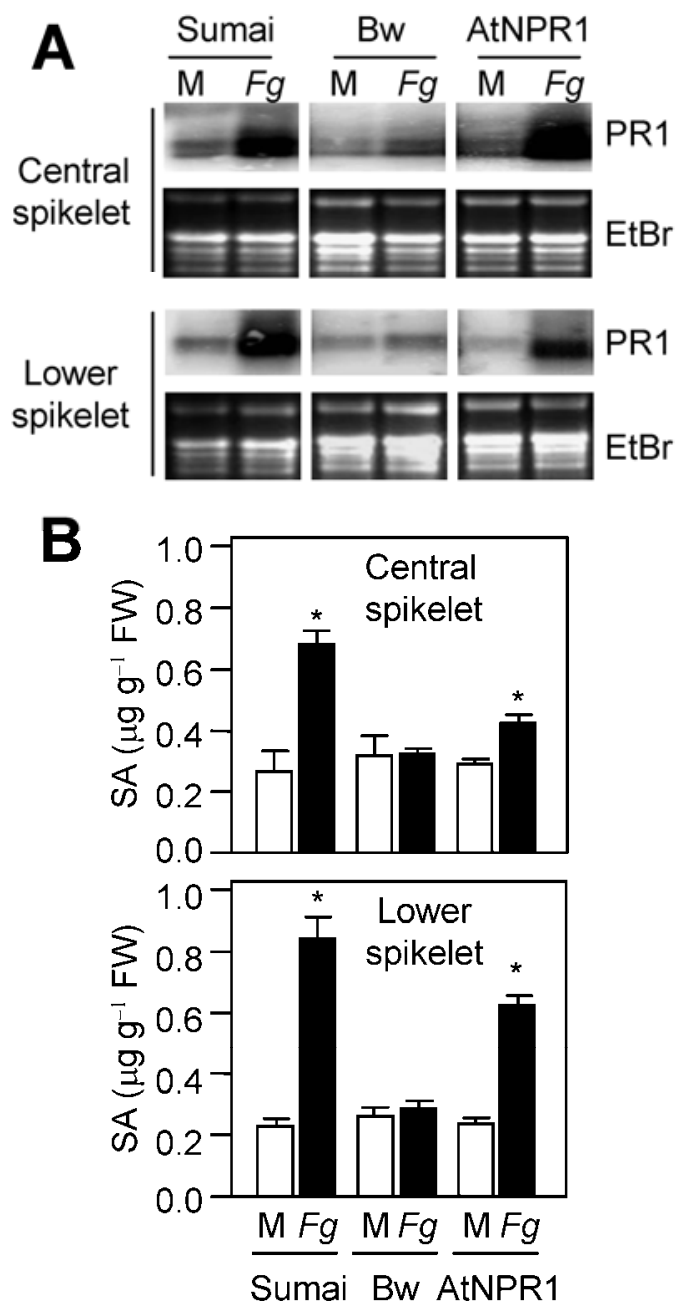

Fig. 1. Activation of a systemic acquired resistance-like mechanism in $F u$ sarium graminearum-infected wheat spikes. A, Pathogenesis-related 1 (PRI) gene expression in $F$. graminearum-infected spikes. RNA blot analysis of PRl expression $24 \mathrm{~h}$ postinfection (hpi) in the central $F$. graminearuminoculated spikelets and lower uninoculated spikelets of the Fusarium head blight (FHB)-resistant wheat 'Sumai-3' (Sumai), the FHB-susceptible 'Bobwhite' (Bw), and FHB-resistant AtNPRl-expressing transgenic plants in the Bobwhite background. A ${ }^{32} \mathrm{P}$-labeled $P R 1$ gene-specific probe was used to monitor $P R 1$ transcript accumulation and ethidium bromide $(\mathrm{EtBr})$ staining was used to monitor quality of RNA and gel loading. B, Salicylic acid (SA) accumulation in $F$. graminearum-infected spikes. SA content was monitored at $24 \mathrm{hpi}$ in the central $F$. graminearum-inoculated spikelets and lower uninoculated spikelets of the wheat Sumai-3 (Sumai), Bobwhite (Bw), and AtNPRl-expressing transgenic plants. SA content is expressed as micrograms per gram fresh weight of spikelets $(n=5)$. Asterisks $(*)$ denote values that are significantly different $(P<0.05 ; t$ test $)$ from the corresponding mock-inoculated plants. compared with the control water-treated spikes (Fig. 2B; Supplementary Fig. S1). SA treatment also resulted in upregulation of the WCII gene, which is also induced by BTH (Gorlach et al. 1996). SA-induced expression of PRI was faster in AtNPRl-expressing wheat than in the nontransgenic wheat Bobwhite. However, AtNPRI expression did not impact the SA-induced expression of WCIl, suggesting that SAinduced upregulation of WCII is likely mediated via a mechanism that is independent of NPR1. Presence of an NPR1-independent SA-regulated processes has also been documented in Arabidopsis (Shah et al. 2001). Soil-drench application of SA and BTH also enhanced FHB resistance in Bobwhite (Figs. 2C and $3 \mathrm{~A}$ ). Levels of DON, a trichothecene toxin produced by $F$. graminearum, was also lower in the $F$. graminearum-inoculated spikes of SA-treated Bobwhite plants than in the control water-treated $F$. graminearum-inoculated plants (Table 1). DON content in SA-treated plants was comparable with that in $F$. graminearum-inoculated spikes of the water-treated AtNPRIexpressing plants. Also, compared with the water-treated $F$. graminearum-inoculated spikes of Bobwhite, 15-acetyl-deoxy-
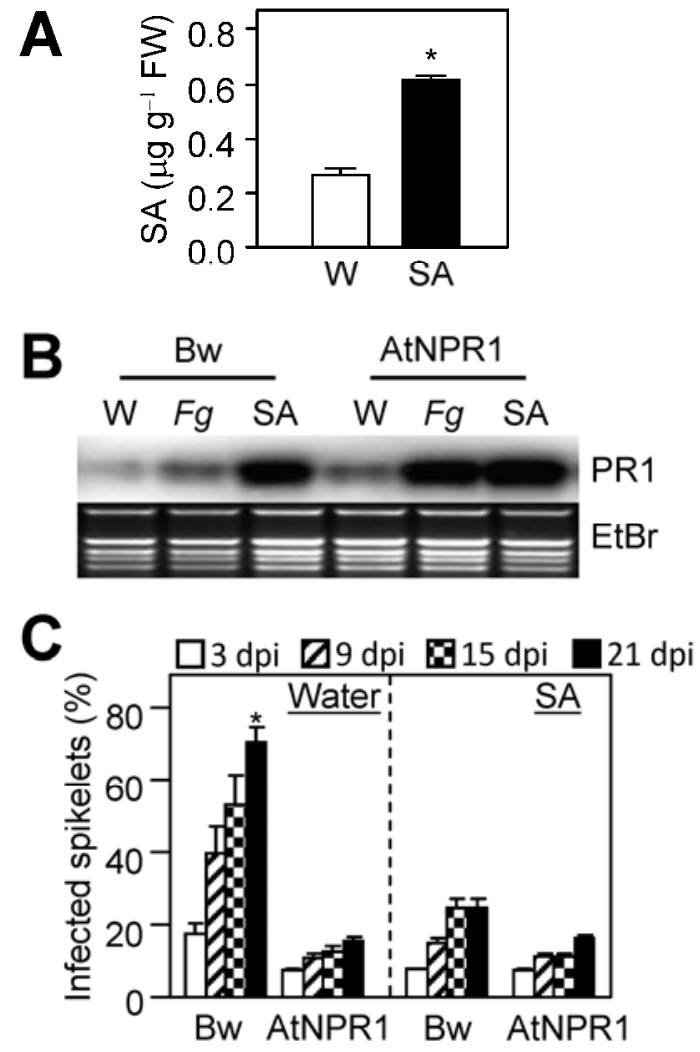

Fig. 2. Exogenous application of salicylic acid (SA) enhances resistance to Fusarium head blight (FHB). A, SA content in spikes of wheat 'Bobwhite' plants that were irrigated with SA $(200 \mu \mathrm{M}) 24 \mathrm{~h}$ prior to sampling. Plants irrigated with water provided the control. SA content is expressed as micrograms per gram fresh weight of spikelets $(n=3)$. Asterisk $(*)$ denotes a bar that is significantly different $(P<0.05 ; t$ test $)$ from the corresponding water-treated control plants. B, RNA blot analysis of pathogenesis-related 1 (PR1) gene expression $24 \mathrm{~h}$ after irrigation of Bobwhite and AtNPR1 plants with SA $(200 \mu \mathrm{M})$ and with water as control. Fusarium graminearum-inoculated spikelets harvested at $24 \mathrm{~h}$ postinfection (hpi) provided additional controls. Ethidium Bromide (EtBr) staining of RNA was used to monitor quality of RNA and gel loading. C, FHB severity in SA-treated plants. FHB disease rating was monitored in Bobwhite and AtNPR1 wheat that were irrigated with SA $(200 \mu \mathrm{M})$ and with water as control $24 \mathrm{~h}$ prior to inoculation of spikes with $F$. graminearum. Disease incidence was recorded at 3, 9, 15, and 21 days postinoculation (dpi) as the percentage of infected spikelets in each spike. At minimum, 20 plants per genotype were tested for each treatment. An asterisk $(*)$ denotes a value that is significantly different $(P<0.05 ; t$ test $)$ from the other treatments at $21 \mathrm{dpi}$. 
nivalenol (15-ADON) was undetectable in the SA-treated, $F$. graminearum-inoculated spikes (Table 1). The above results confirm that activation of SA signaling can curtail FHB infection and mycotoxin accumulation in wheat.
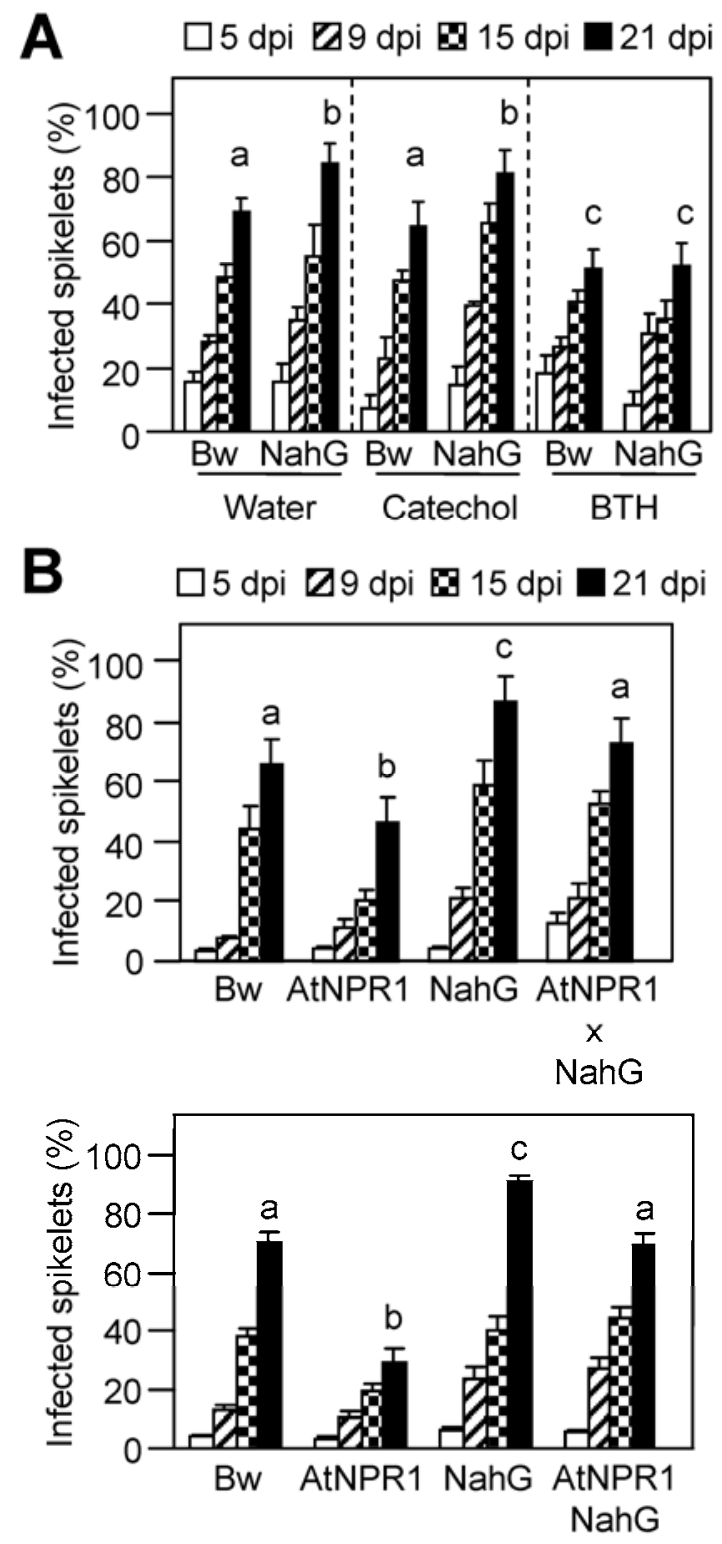

Fig. 3. Salicylic acid (SA) is required for basal resistance to Fusarium head blight (FHB) in wheat. A, FHB severity in wheat 'Bobwhite' and in transgenic plants expressing the salicylate hydroxylase encoding $N a h G$ gene. FHB disease rating was monitored in plants that were irrigated with catechol $(200 \mu \mathrm{M})$ or benzo $(1,2,3)$, thiadiazole-7 carbothioic acid $S$ methyl ester $(\mathrm{BTH})(200 \mu \mathrm{M})$ and with water as control $24 \mathrm{~h}$ prior to Fusarium graminearum inoculation. Disease incidence was recorded at 5 , 9, 15, and 21 days postinoculation (dpi) as the percentage of infected spikelets in each spike. At minimum, 20 plants per genotype were tested for each treatment. Different letters above bars indicate values at $21 \mathrm{dpi}$ that are significantly different $(P<0.05)$ from each other as determined by general linear models analysis of variance (GLM/ANOVA). B, FHB severity in wheat plants co-expressing AtNPRI and NahG. Upper panel: FHB disease rating was monitored in wheat Bobwhite $(\mathrm{Bw})$ and transgenic AtNPR1 or NahG plants, and in $\mathrm{F}_{1}$ plants derived from a cross between an AtNPR1 and an NahG plant. Lower panel: FHB disease rating was monitored in wheat Bobwhite $(\mathrm{Bw})$ and transgenic plants expressing AtNPR1 or $N a h G$, and AtNPRl and $N a h G$ co-expressing $\mathrm{F}_{2}$ progeny derived from AtNPR1/NahG $\mathrm{F}_{1}$ hybrid plants. Disease incidence was recorded at 5, 9, 15 , and $21 \mathrm{dpi}$ as the percentage of infected spikelets in each spike. Different letters above bars indicate values at 21 dpi that are significantly different $(P<0.05)$ from each other as determined by GLM/ANOVA.
$\mathrm{SA}$ is required for basal resistance to $\mathrm{FHB}$ in wheat.

To determine whether SA is important for basal resistance against FHB, the bacterial NahG gene-encoded salicylate hydroxylase was stably expressed under control of the Zea mays Ubiquitin (Ubi) gene promoter in transgenic wheat. The NahG transcript was expressed and SA content significantly lower in NahG transgenic wheat compared with the nontransgenic Bobwhite plants (Supplementary Fig. S2A and B). FHB severity

Table 1. Mycotoxin content in Fusarium graminearum-inoculated wheat ${ }^{\mathrm{a}}$

\begin{tabular}{lccc}
\hline Genotype & Treatment $^{\mathbf{b}}$ & DON (ppm) & 15-ADON $(\mathbf{p p m})$ \\
\hline Bobwhite & Water & $6.37 \pm 1.76$ & $0.22 \pm 0.04$ \\
& SA & $1.93 \pm 0.47$ & nd \\
AtNPR1 & Water & $0.57 \pm 0.39$ & nd \\
& SA & nd & nd \\
\hline
\end{tabular}

${ }^{\mathrm{a}} \mathrm{DON}=$ deoxynivalenol, 15 -ADON $=15$-acetyl-deoxynivalenol, and $\mathrm{nd}=$ not detected.

${ }^{\mathrm{b}}$ Plants were irrigated with salicylic acid (SA) $(200 \mu \mathrm{M})$ and water as control for $24 \mathrm{~h}$ prior to $F$. graminearum inoculation.

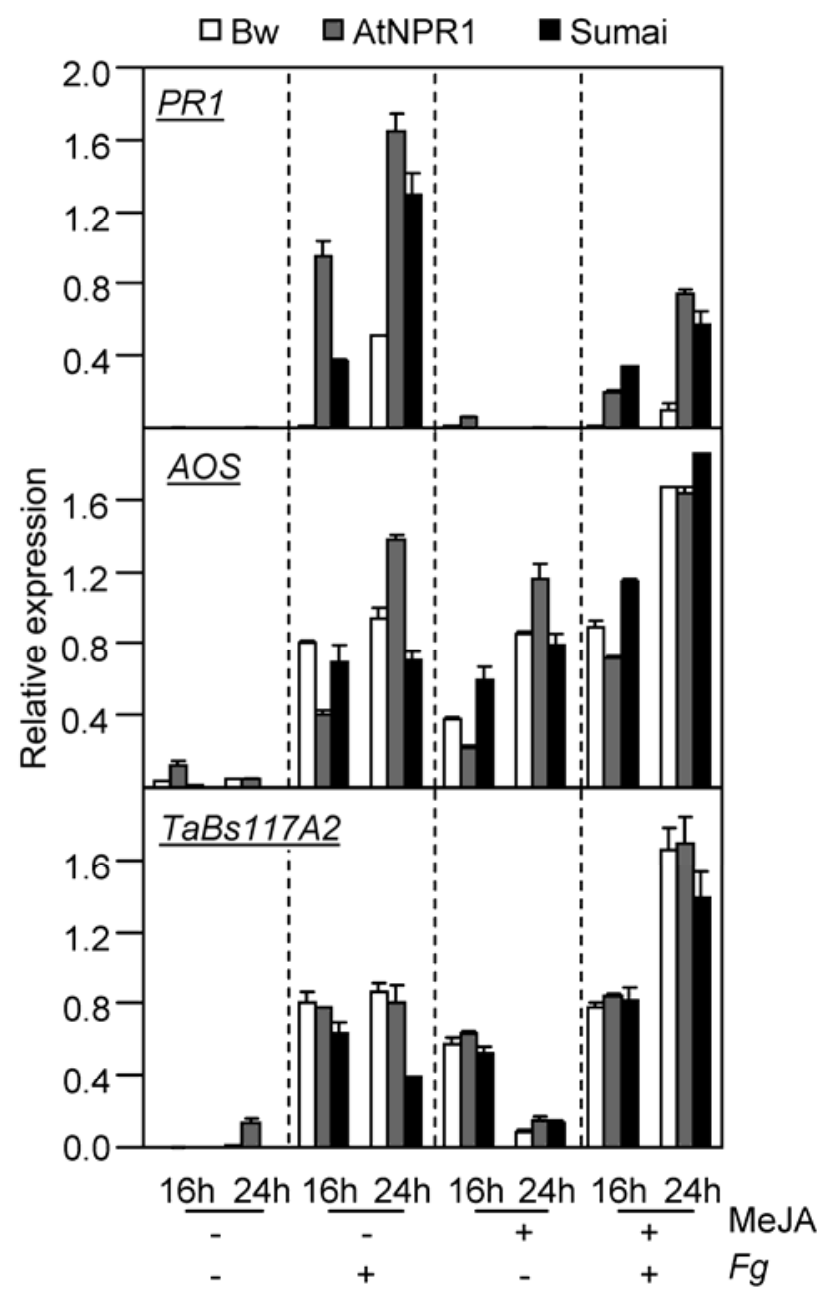

Fig. 4. Jasmonic acid (JA) signaling is activated in wheat spikes in response to Fusarium graminearum infection. Semiquantitative reverse-transcriptase polymerase chain reaction analysis was performed on the salicylic acid (SA)-responsive pathogenesis-related $1(P R 1)$ and the JA-responsive $A O S$ and TaBs117A2 genes in spikes of $F$. graminearum-inoculated wheat 'Bobwhite' (Bw), AtNPR1 wheat, and 'Sumai-3' (Sumai) plants that were exposed to methyl-jasmonic acid (MeJA) vapors in an enclosed chamber for a 24-h period immediately prior to fungal inoculation. Gene expression was monitored at 16 and $24 \mathrm{~h}$ postinfection with $F$. graminearum and as a control in mock-inoculated plants. Each bar represents the relative expression of the genes compared with the Actin control. 
was higher in NahG wheat compared with nontransgenic Bobwhite plants (Fig. 3A and B). Because catechol, the product of salicylate hydroxylase action on SA, did not enhance FHB severity in Bobwhite (Fig. 3A), the impact of $N a h G$ expression on FHB resistance is likely due to the reduced SA content in NahG plants. To test this further, we determined whether application of BTH, which is a synthetic functional analog of SA but not a substrate for the $N a h G$-encoded salicylate hydroxylase, could restore FHB resistance in NahG wheat. BTH (200 $\mu \mathrm{M})$ was applied as a soil drench to NahG wheat and as a control to nontransgenic Bobwhite plants $24 \mathrm{~h}$ prior to inoculation of spikes with $F$. graminearum. BTH application reduced FHB severity in NahG plants and also enhanced FHB resistance in nontransgenic Bobwhite plants (Fig. 3A). Thus, we conclude that SA signaling has a critical role in basal resistance against FHB.

To determine whether SA is also important for the heightened FHB resistance observed in AtNPRl-expressing wheat, plants simultaneously expressing both $N a h G$ and AtNPRl were generated by crossing NahG wheat with AtNPR1 wheat.
Both of these transgenics are in the Bobwhite background. FHB disease severity was monitored in spikes of NahG/AtNPR $1 F_{1}$ hybrids and in the $F_{2}$ progeny that expressed both $N a h G$ and AtNPRl. Compared with the AtNPR1 wheat, presence of $N a h G$ attenuated the AtNPRl-promoted FHB resistance in the NahG/AtNPR1 $\mathrm{F}_{1}$ hybrids and $\mathrm{F}_{2}$ progeny plants that expressed both $N a h G$ and AtNPRl (Fig. 3B), thus confirming that $\mathrm{SA}$ is required for the heightened FHB resistance observed in AtNPR1 wheat.

\section{A complex interaction between $\mathrm{SA} / \mathrm{NPR} 1$ and}

JA signaling in $\boldsymbol{F}$. graminearum-infected wheat.

A previous study indicated that daily spray and point applications of MeJA for three consecutive days to the spikelets of an FHB-susceptible wheat landrace, Y1193-6, enhanced resistance against FHB (Li and Yen 2008). Furthermore, expression of several JA-responsive genes was upregulated in $F$. graminearum-inoculated wheat ( $\mathrm{Li}$ and Yen 2008), thus suggesting the involvement of JA signaling in wheat interaction with $F$. graminearum (Li and Yen 2008). In Arabidopsis, JA contrib-
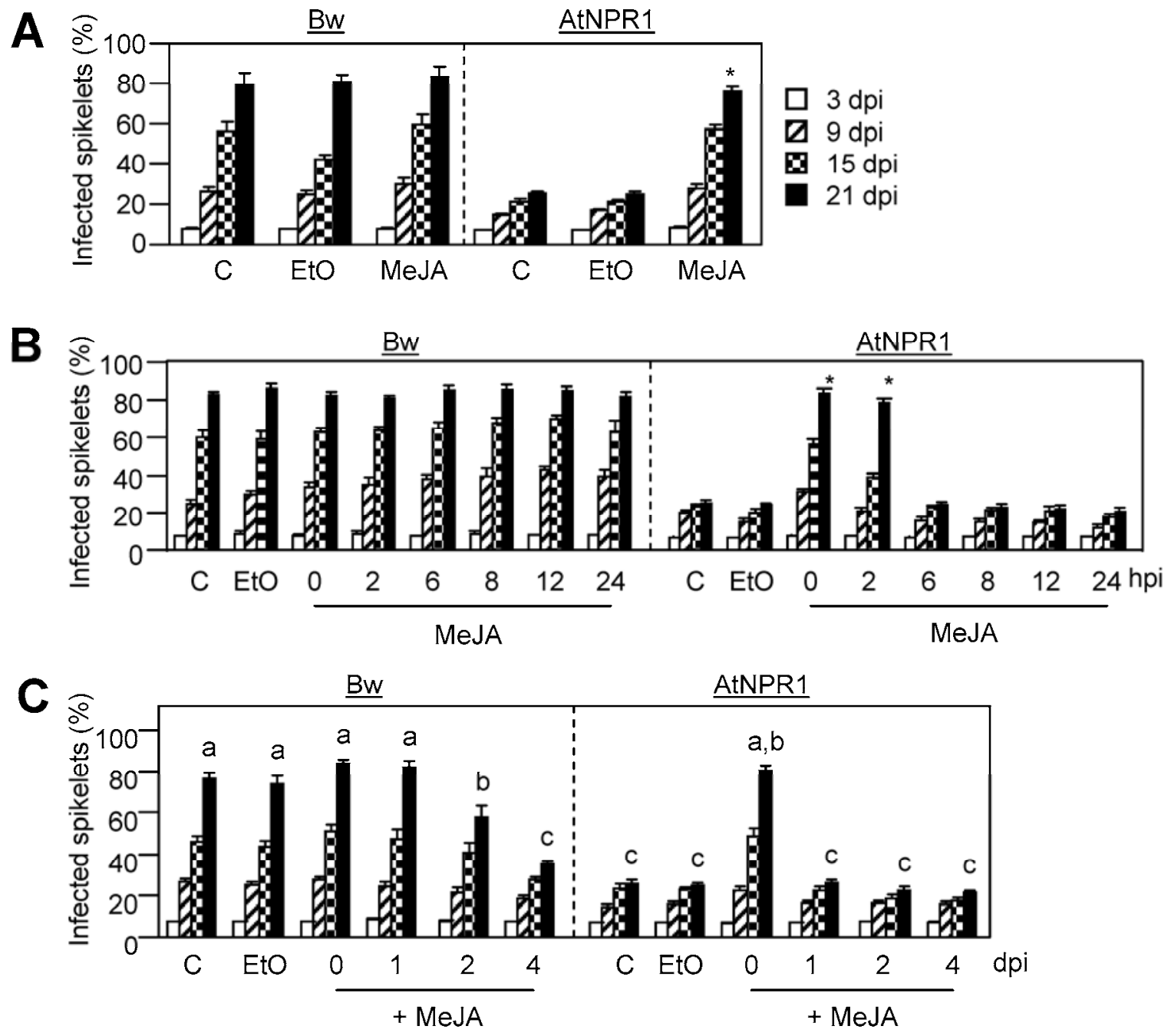

Fig. 5. Impact of methyl-jasmonic acid (MeJA) treatment on Fusarium head blight (FHB) severity in wheat. A, Comparison of FHB incidence in wheat 'Bobwhite' (Bw) and AtNPR1 plants that were exposed to MeJA vapors dissolved in 0.1\% ethanol (EtO) in an enclosed chamber for a 24-h period prior to Fusarium graminearum inoculation. B, FHB incidence in Bw and AtNPR1 plants that were exposed to MeJA vapors for a 24-h period beginning at 0, 2, 6, 8, 12, and $24 \mathrm{~h}$ after fungal inoculation (hpi). C, FHB incidence in Bw and AtNPR1 plants that were exposed to MeJA (vapors) for a 24-h period beginning at 0, 1,2 , and 4 days after fungal inoculation (dpi). A to $\mathbf{C}$, Plants that were exposed to $0.1 \%$ EtO or plants that did not receive any chemical treatment (C) provided controls. Central spikelets of each spike were inoculated. Disease incidence was recorded at 3,9,15, and 21 dpi as the percentage of diseased spikelets in each spike. A and $\mathbf{B}$, Asterisks $(*)$ denote bars that are significantly different from plants that did not receive chemical treatment $(P<0.05$; general linear models [GLM] at $21 \mathrm{dpi}$. C, Different letters above bars indicate values at $21 \mathrm{dpi}$ that are significantly different $(P<0.05)$ from each other as determined by GLM analysis of variance. 
utes to defense against $F$. graminearum, as well (Makandar et al. 2010). However, genetic studies indicated that, during early stages of infection, jasmonates suppressed the activation of defense signaling through NPRI. This effect of JA was very prominent in Arabidopsis plants that were engineered to overexpress NPRl (Makandar et al. 2010). Therefore, we tested whether pretreatment with MeJA similarly attenuated AtNPRI expression-conferred enhanced FHB resistance in wheat. Exposure of AtNPR1-expressing wheat to MeJA vapors for a 24-h period prior to $F$. graminearum inoculation resulted in expression of the JA-responsive genes $A O S$ and TaBs117A2, thus confirming the activation of JA signaling (Fig. 4; Supplementary Fig. S3). MeJA application also attenuated FHB resistance in AtNPR1 wheat (Fig. 5A). Suppression of AtNPR1-conferred FHB resistance was also observed when MeJA was applied for a 24-h period beginning at the time of fugal inoculation $(0 \mathrm{~h})$ (Fig. 5B). Fungal infection-induced expression of $P R I$ was weaker in the MeJA-treated than ethanol $(0.1 \%)$-treated AtNPR1 plants (Fig. 4), suggesting that MeJA pretreatment attenuated the activation of SA signaling in $F$. graminearuminfected spikelets. A similar attenuation of $P R I$ expression by MeJA treatment was also observed in the spikes of $F$. graminearum-challenged nontransgenic Bobwhite and Sumai-3 plants (Fig. 4). The ability of JA to attenuate activation of SA signaling (Pieterse et al. 2009) may explain the attenuation of AtNPRl-conferred FHB resistance in wheat. To determine whether or not this is the case, we treated AtNPR1 wheat with MeJA for a 24-h period beginning $2 \mathrm{~h}$ postinoculation (hpi) with $F$. graminearum. Expression of the SA-responsive PRI gene was already induced to high levels by $6 \mathrm{hpi}$ in the spikes of AtNPR1 wheat (Supplementary Fig. S4). In contrast to MeJA exposure beginning at the time of fungal inoculation (0 h), which attenuated AtNPRl-determined FHB resistance, MeJA exposure beginning at $6 \mathrm{hpi}$ and beyond did not attenuate the AtNPRl-determined enhanced FHB resistance (Fig. $5 \mathrm{~B})$. On the other hand, exposure of nontransgenic Bobwhite to MeJA vapors did not exhibit any significant enhancement of disease severity when applied during these early time points (Fig. 5A and B). However, when applied for a 24-h period beginning 2 days postinoculation (dpi) and later, MeJA enhanced FHB resistance in Bobwhite plants (Fig. 5C). These results suggest that, as in Arabidopsis, a complex interaction between $\mathrm{SA} / N P R 1$ and JA signaling regulates basal resistance against FHB.

\section{DISCUSSION}

Prior to this study, the role of SA in wheat defense against FHB was unclear. Here, we show that SA, when applied as a soil drench, which results in an increase in SA content of wheat spikes (Fig. 2A), effectively enhanced PRI expression and resistance to FHB (Fig. 2B and C) and curtailed DON and 15-ADON accumulation (Table 1). BTH applied as a soil drench also promoted resistance to FHB (Fig. 3A). Studies of NahG wheat provide genetic evidence confirming the importance of SA signaling in basal resistance against $F$. graminearum infection. FHB severity was higher in NahG wheat compared with nontransgenic Bobwhite plants (Fig. 3A and B). Furthermore, resistance to $\mathrm{FHB}$ in NahG wheat was restored by BTH application (Fig. 3A). Expression of NahG also attenuated the FHB resistance conferred by AtNPRI expression (Fig. 3B). These results strongly indicate that, as in Arabidopsis, SA signaling is a critical component of basal resistance in wheat against $F$. graminearum.

Although we show that SA and BTH application induced $P R 1$ expression and enhanced FHB resistance in wheat (Figs. $2 \mathrm{~B}$ and $\mathrm{C}$ and $3 \mathrm{~A}$ ), previous studies did not observe a similar effect of SA and BTH on curtailing FHB infection (Li and Yen 2008; Yu and Muehlbauer 2001). The most likely explanation for this is the difference in how SA and BTH were applied in these studies. SA and BTH were applied as a soil drench in our experiments (Figs. 2A to C and 3A; Table 1). Soil-drench application of SA increased SA content in wheat spikes (Fig. 2A). Furthermore, soil-drench application of SA resulted in robust induction of $P R I$ and $W C I 1$ gene expression in wheat spikes (Fig. 2B), thus confirming the effectiveness of this application method in systemically activating SA signaling. By contrast, SA applied by spraying spikes to run-off did not enhance FHB resistance ( $\mathrm{Li}$ and Yen, 2008). The levels of SA and gene expression were not monitored in spikes of plants sprayed with SA and, hence, it is unclear whether SA signaling was activated in those experiments ( $\mathrm{Li}$ and Yen 2008). Similarly, although spray treatment with BTH induced $W C I$ gene expression in wheat spikes, it was unable to induce $P R 1$ expression ( $\mathrm{Yu}$ and Muehlbauer 2001), thus suggesting that spraying BTH on wheat spikes may not have the same physiological effect as irrigating wheat plants with BTH. AtNPRl expression in wheat enhanced the effect of soil-drench-applied SA on stimulating $P R I$ expression without impacting the SA-induced expression of $W C I 1$, thus suggesting differences in the regulation of $W C I 1$ and $P R 1$ expression by SA and, likely, BTH as well. It is plausible that spray application, unlike irrigation, does not build up high enough levels of these chemicals in spikes to stimulate PRI expression compared with WCII gene expression. Indeed, WCI1 expression was more sensitive to SA than PRI expression even when SA was applied by the soil-drench method. It is also possible that spray treatment of wheat spikes does not deliver these chemicals to the tissues in which SA- or BTHmediated defenses against $F$. graminearum are exerted and PRI expressed, compared with tissues in which $W C I$ genes may be expressed. Whether PRI and the WCII genes are expressed in the same tissues is not known. At this stage, we cannot rule out any contribution of genotype differences to this discrepancy, as well.

In Arabidopsis, both SA/NPRI and JA signaling are required for the full level of basal resistance against $F$. graminearum (Makandar et al. 2010). However, JA signaling has a dichotomous involvement in Arabidopsis interaction with F. graminearum, contributing to attenuation of SA signaling during the early stages of infection and promotion of defense against $F$. graminearum during later stages of infection (Makandar et al. 2010). Similarly, in wheat, we find that exposure to MeJA vapors during later stages of infection enhanced FHB resistance in Bobwhite (Fig. 5C). However, when applied prior to fungal inoculation or early during infection, we did not observe a protective effect of MeJA in Bobwhite (Fig. 5A and B). Quite to the contrary, exposure to MeJA vapors either prior to fungal inoculation or during the early stages of fungal infection attenuated AtNPRl-conferred FHB resistance (Fig. 5A and $B$ ). This suggests that, during the early stages of infection, JA signaling attenuates $\mathrm{SA} / N P R l$-determined defense signaling. Indeed, $P R l$ expression in response to $F$. graminearum infection was attenuated in spikes of plants that were pretreated with MeJA (Fig. 4). It is likely that, during the early stages of $F$. graminearum infection when there is no evidence of necrotrophy (Brown et al. 2010; Goswami and Kistler 2004), activation of SA signaling curtails $F$. graminearum infection and, during the later stages of interaction, JA-regulated defenses target the necrotrophic phase of the fungal life cycle. Indeed, Ding and associates (2011) recently showed that SA increases, which peaked at $3 \mathrm{hpi}$, preceded the peak increase in JA level observed at 12 hpi in $F$. graminearum-inoculated spikes of the FHB-resistant wheat germplasm 'Wangshuibai'. By contrast, basal levels of SA were lower in the FHB-susceptible 
'Meh0106' mutant that was identified from a population of ethylmethane sulphonate-mutagenized Wangshuibai seed. $F$. graminearum infection-associated increase in SA was also slower in spikes of the Meh0106 mutant than Wangshuibai (Ding et al. 2011). Furthermore, expression of the wheat homologue of phenylalanine ammonia lyase $(P A L)$, which is involved in the synthesis of several secondary metabolites including SA, as well as expression of wheat homologues of enhanced disease susceptibility 1 (EDS1) and NPR1, which are associated with SA signaling in Arabidopsis, increased faster in Wangshuibai than in the Meh0106 mutant. This increase in PAL, EDS1, and NPR1 expression in Wangshuibai preceded the upregulation of oxophytodienoic acid reductase 3 , which encodes a protein involved in JA synthesis (Ding et al. 2011), thus supporting our suggestion that, compared with JA, SA signaling functions earlier during the infection to limit $F$. graminearum infection.

By contrast to our results with Bobwhite plants exposed to MeJA vapors (Fig. 5A), Li and Yen (2008) showed that repeated spray and point applications of MeJA over a 3-day period prior to fungal inoculation of the FHB-susceptible wheat landrace Y1193-6 curtailed FHB severity. Taken together, the observations of $\mathrm{Li}$ and Yen (2008) and results presented here indicate that hyperactivation of either JA or the SA pathway has the ability to override the need of the other for controlling FHB. It is also plausible that the overall individual contribution of SA and JA signaling to wheat defense against FHB is determined by the plant genotype, thus contributing to the differences between results presented here and by $\mathrm{Li}$ and Yen (2008). Clearly, the role of JA signaling in wheat interaction with $F$. graminearum and its interaction with SA signaling needs to be genetically validated.

In conclusion, we have demonstrated that SA signaling contributes to basal resistance against FHB in wheat. However, the activation of SA signaling can be constrained by the simultaneous or prior activation of JA signaling. Despite this antagonism, JA also contributes to resistance against $F$. graminearum. This role of JA is most evident from the genetic studies conducted in Arabidopsis (Makandar et al. 2010). Thus, any effort to target the SA- and JA-regulated mechanisms for controlling FHB in wheat should consider the complexity in interaction between $\mathrm{SA} / N P R 1$ and JA signaling and the impact of this cross-talk on the host's ability to curtail $F$. graminearum infection.

\section{MATERIALS AND METHODS}

\section{Plant growth conditions.}

The AtNPRl-expressing transgenic wheat line in the spring wheat Bobwhite background was described earlier (Makandar et al. 2006). Wheat plants were grown in a greenhouse under a temperature regime of 21 and $18^{\circ} \mathrm{C}$ during the day and night, respectively. Wheat seed were grown in autoclaved composedpeat-based planting mixture (Premier Pro Mix-BX; Premier Tech Horticulture, Rivière-du-Loup, Canada), three seeds per gallon-capacity pot.

\section{Fungal growth conditions.}

$F$. graminearum isolate Z-3639 was cultured in half-strength potato dextrose agar (Difco Laboratories, Detroit) at $22^{\circ} \mathrm{C}$. Fungal sporulation was induced as described earlier (Makandar et al. 2006).

\section{Fungal inoculation of wheat and FHB disease rating.}

Wheat spikes of greenhouse plants at anthesis were inoculated with an F. graminearum spore suspension containing 30,000 spores $\mathrm{ml}^{-1}$. The central spikelets, one on either side of the rachis, were inoculated with $10 \mu \mathrm{l}$ of $F$. graminearum spore suspension (300 spores per spikelet). Three spikes per plant were inoculated. The inoculated spikes were covered with moistened zip-lock bags. The bags were removed 3 dpi. The plants were evaluated for FHB disease at periodic intervals as percent spikelets infected with FHB. The experiments to analyze FHB incidence on wheat plants were repeated three times, with three replications each. The significance of the data was tested by $t$ test and analysis of variance using the general linear models procedure available in Minitab (version 15; Minitab Inc., State College, PA, U.S.A.) as indicated.

\section{Plasmids and wheat transformation.}

The Ubi:NahG chimeric gene construct (plasmid pVJN01) was generated by cloning the coding sequence for $P$. putida $N a h G$ gene under control of the maize $U b i$ gene promoter and nopaline synthase terminator from Agrobacterium tumefaciens in the pJS406 vector backbone (Makandar et al. 2006). The maize $U b i$ intron was included at the start of the NahG coding region to enhance stability of the encoded transcript. Ubi:NahG and a plasmid containing the Ubi:bar construct (pAHC20) (Christensen and Quail 1996) were co-bombarded into immature wheat embryos. The $B A R$ gene confers resistance to the herbicide glufosinate (Liberty; Bayer Crop Sciences, Research Trianle, NC, U.S.A.). Regeneration of wheat embryos was performed using a previously described protocol (Altpeter et al. 1996) as modified by Anand and associates (2003b). The NahG wheat line did not exhibit any discernible detrimental growth or developmental phenotypes in the greenhouse conditions used in this study.

\section{Molecular analysis of transgenic NahG plants.}

The DNA from leaves of transgenic wheat plants was extracted as previously described (Makandar et al. 2006). Genomic polymerase chain reaction (PCR) was used to monitor the presence of Ubil:NahG chimera. NahG-specific oligonucleotide primers NahG-F1 (5'-TCACCTCCCAGAAGGTATCG-3') and NahG-R1 (5'-GAGATGAAAGCCACCACGTT-3') were used to amplify a 450-bp fragment of the Ubil:NahG chimera. Primers for wheat Tubulin Tub-F (5'-GACATCAACATTCAG AGCACCATC-3') and Tub-R (5'-ATCTGTGCCTTGACCGT ATCAGG-3'), which amplify a 409-bp fragment, were used as an internal control.

\section{RNA isolation and analysis.}

RNA was extracted from $500 \mathrm{mg}$ of spike tissue using acid guanidinium thiocyanate-phenol-chloroform solution (Chomczynski and Sacchi 1987). The isolated RNA was quantified using a spectrophotometer at $230 \mathrm{~nm}$. RNA was denatured (10 to $15 \mu \mathrm{g}$ ), run on formaldehyde-agarose gels, and transferred to Hybrid-N+ Nylon membrane (GE Healthcare, Piscataway, NJ, U.S.A.). RNA blots were probed with a ${ }^{32} \mathrm{P}$-labeled DNA fragment of FHB-inducible wheat $P R 1$ gene amplified from the wheat $P R I$ clone (GenBank accession number DT045069). The ethidium-bromide-stained RNA serves as the internal control. Gene expression was also studied using reverse-transcriptase (RT)-PCR. For RT-PCR analysis, RNA was purified with an RNA purification kit (Qiagen Inc., Valencia, CA, U.S.A.). Actin and Tubulin expression provided the control for RT-PCR. Conditions used for RT-PCR studies are as previously described (Makandar et al. 2006). For quantitative analysis, the RT-PCR products were resolved on a $1.5 \%$ agarose gel containing ethidium bromide. The relative intensities of the RT-PCR products were obtained using the histogram function in GIMP 2.6 and then normalized to the expression of Actin. Gene-specific primers used for monitoring $P R l$ expression were PR1-F (5'CGCAGAACTCGCCGCAGGAC-3') and PR1-R (5'-GCAGA CGACACGAGCACAGC-3'), for AOS (GenBank accession number: AY196004.1) were AOS-F (5'-ACGTCGACAAGGTC 
GAGAAG-3') and AOS-R (5'-GGGCAAGATTGCGAAAAGT A-3'), for the JA-inducible putative lipase encoding TaBs $117 A 2$ (Lu et al. 2005) were LIPASE-F (5'-CACAAAAATATCGACC CACCAC-3') and LIPASE-R (5'-ACTGGGTATTCGTCTGTC AGC-3'), and for Actin were Actin8-F (5'-ATGAAGATTAAGG TCGTGGCA- $3^{\prime}$ ) and Actin8-R (5'-TCCGAGTTTGAAGAGGC TAC- $\left.3^{\prime}\right)$. Sequences of primers used for monitoring $N a h G$ and Tubulin expression are detailed above.

\section{Chemical treatment of plants.}

Stock solutions of $10 \mathrm{mM}$ sodium-salicylate (Sigma-Aldrich, St. Louis), BTH (50\% active ingredient), and catechol (SigmaAldrich) were prepared in water and diluted to a final concentration of $200 \mu \mathrm{M}$. Pretreatment of wheat with SA, BTH, or catechol in greenhouse trials was done by irrigating the potted plants 1 day before $F$. graminearum inoculation. Control plants were irrigated with water before inoculating with $F$. graminearum. MeJA (Sigma-Aldrich) stock solution (1 mM) was prepared in $0.1 \%$ ethanol and the stock solution diluted in $0.1 \%$ ethanol to a working solution of $200 \mu \mathrm{M}$. Wheat plants were exposed to MeJA vapors for $24 \mathrm{~h}$ in a plexiglass chamber. Control plants were treated with $0.1 \%$ ethanol and water in a similar manner. Plants were treated by placing potted plants in plexiglass chambers (48-liter volume) containing cotton plugs soaked with $1 \mathrm{ml}$ of MeJA solution $(200 \mu \mathrm{M})$ in $0.1 \%$ ethanol. The moistened cotton plugs were placed in a petri plate in the center of the chamber.

\section{SA quantification.}

A gas chromatography-mass spectrometry method (Schmelz et al. 2004), was adapted to quantify SA and JA content in wheat spikes (Makandar et al. 2010). In all, 100 to $200 \mathrm{mg}$ fresh weight of spikelet tissue was harvested from wheat plants and frozen immediately in liquid nitrogen. Five replications per each treatment were used for the estimation of total SA (SA + SA-glucoside) and JA content. Quantification of total SA in NahG lines was done using a modified biosensor strain of an Acinetobacter sp., ADP1. Leaves were extracted in fresh Luria-Bertani medium $(2.5 \mathrm{ml} / \mathrm{g}$ of leaf $)$, further sonicated on ice for $5 \mathrm{~min}$, and centrifuged. The supernatant was used for SA estimation using the biosensor strain as described previously (Huang et al. 2005, 2006).

\section{Mycotoxin analysis.}

DON and ADON content in wheat grain was determined as previously described (Fuentes et al. 2005; Mirocha et al. 1998).

\section{ACKNOWLEDGMENTS}

We thank A. Sparks and R. Welti at the Kansas Lipidomics Research Center, Kansas State University for mass spectrometry analysis. This material is based upon work supported by the United States Department of Agriculture, under agreement number 59-0790-8-060 (to J. Shah and H. N. Trick) and 59-0206-9-074 (to Y. Dong) as cooperative projects with the U.S. Wheat \& Barley Scab Initiative. Kansas Lipidomics Research Center was supported by National Science Foundation (EPS 0236913, MCB 0455318, DBI 0521587), Kansas Technology Enterprise Corporation, KIDeA Networks of Biomedical Research Excellence (INBRE) of National Institute of Health (P20RR16475), and Kansas State University. V. J. Nalam was supported by a Graduate Research Fellowship from the College of Arts and Science at the University of North Texas. This article is contribution number 12-091-J from the Kansas Agricultural Experimental Station, Kansas State University, Manhattan.

\section{LITERATURE CITED}

Adie, B. A. T., Pérez-Pérez, J., Pérez-Pérez, M. M., Godoy, M., SánchezSerrano, J.-J., Schmelz, E. A., and Solano, R. 2007. ABA is an essential signal for plant resistance to pathogens affecting JA biosynthesis and the activation of defenses in Arabidopsis. Plant Cell 19:1665-1681.

Altpeter, F., Vasil, V., Srivastava, V., Stöger, E., and Vasil, I. K. 1996. Accelerated production of transgenic wheat (Triticum aestivum L.) plants. Plant Cell Rep. 16:12-17.

Anand, A., Schmelz, E. A., and Muthukrishnan, S. 2003a. Development of a lesion-mimic phenotype in a transgenic wheat line overexpressing genes for pathogenesis-related (PR) proteins is dependent on salicylic acid concentration. Mol. Plant-Microbe Interact. 16:916-925.

Anand, A., Zhou, T., Trick, H. N., Gill, B. S., Bockus, W. W., and Muthukrishnan, S. 2003b. Greenhouse and field testing of transgenic wheat plants stably expressing genes for thaumatin-like protein, chitinase and glucanase against Fusarium graminearum. J. Exp. Bot. 54:11011111.

Bai, G.-H., and Shaner, G. 2004. Management and resistance in wheat and barley to Fusarium head blight. Annu. Rev. Phytopathol. 4:135-161.

Balbi, V., and Devoto, A. 2008. Jasmonate signalling network in Arabidopsis thaliana: Crucial regulatory nodes and new physiological scenarios. New Phytol. 177:301-318.

Berrocal-Lobo, M., Molina, A., and Solano, R. 2002. Constitutive expression of ETHYLENE-RESPONSE-FACTOR1 in Arabidopsis confers resistance to several necrotrophic fungi. Plant J. 29:23-32.

Brown, N. A., Urban, M., van de Meene, A. M. L., and Hammond-Kosack, K. E. 2010. The infection biology of Fusarium graminearum: Defining the pathways of spikelet to spikelet colonisation in wheat ears. Fungal Biol. 114:555-571.

Browse, J. 2009. Jasmonate passes muster: A receptor and targets for the defense hormone. Annu. Rev. Plant Biol. 60:183-205.

Buerstmayr, H., Ban, T., and Anderson, J.A. 2009. QTL mapping and marker-assisted selection for Fusarium head blight resistance in wheat: A review. Plant Breed. 128:1-26.

Cao, H., Bowling, S. A., Gordon, A. S., and Dong, X. 1994. Characterization of an Arabidopsis mutant that is nonresponsive to inducers of systemic acquired resistance. Plant Cell 6:1583-1592.

Chaturvedi, R., and Shah, J. 2007. Salicylic acid in plant disease resistance. Pages 335-370 in: Salicylic Acid-A Plant Hormone. S. Hayat and A. Ahmad, eds. Springer, Dordrecht, The Netherlands.

Chaturvedi, R., Krothapalli, K., Makandar, R., Nandi, A., Sparks, A. A., Roth, M. R., Welti, R., and Shah, J. 2008. Plastid $\omega 3$-fatty acid desaturase-dependent accumulation of a systemic acquired resistance inducing activity in petiole exudates of Arabidopsis thaliana is independent of jasmonic acid. Plant J. 54:106-117.

Chen, X., Steed, A., Harden, C., and Nicholson, P. 2006. Characterization of Arabidopsis thaliana-Fusarium graminearum interactions and identification of variation in resistance among ecotypes. Mol. Plant Pathol. 7:391-403.

Chen, X., Steed, A., Travella, S., Keller, B., and Nicholson, P. 2009. Fusarium graminearum exploits ethylene signalling to colonize dicotyledonous and monocotyledonous plants. New Phytol. 182:975-983.

Chomczynski, P., and Sacchi, N. 1987. Single-step method of RNA isolation by acid guanidinium thiocyanate-phenol-chloroform extraction. Anal. Biochem. 162:156-159.

Christensen, A. H., and Quail, P. H. 1996. Ubiquitin promoter-based vectors for high-level expression of selectable and/or screenable marker genes in monocotyledonous plants. Transgenic Res. 5:213-218.

Clarke, J. D., Volko, S. M., Ledford, H., Ausubel, F. M., and Dong, X. 2000. Roles of salicylic acid, jasmonic acid, and ethylene in cpr-induced resistance in Arabidopsis. Plant Cell 12:2175-2190.

Cuzick, A., Lee, S., Gezan, S., and Hammond-Kosack, K. E. 2008. NPR1 and EDS11 contribute to host resistance against Fusarium culmorum in Arabidopsis buds and flowers. Mol. Plant Pathol. 9:697-704.

Delaney, T. P., Friedrich, L., and Ryals, J. A. 1995. Arabidopsis signal transduction mutant defective in chemically and biologically induced disease resistance. Proc. Natl. Acad. Sci. U.S.A. 92:6602-6606.

Ding, L., Xu, H., Yi, H., Yang, L., Kong, Z., Zhang, L., Xue, S., Jia, H., and $\mathrm{Ma}, \mathrm{Z}$. 2011. Resistance to hemi-biotrophic $F$. graminearum infection is associated with coordinated and ordered expression of diverse defense signaling pathways. PLoS One 6:e19008. Published online.

Durrant, W. E., and Dong, X. 2004. Systemic acquired resistance. Annu. Rev. Phytopathol. 42:185-209.

Ferrari, S., Plotnikova, J. M., De Lorenzo, G., and Ausubel, F. M. 2003. Arabidopsis local resistance to Botrytis cinerea involves salicylic acid and camalexin and requires EDS4 and PAD2, but not SID2, EDS5 or PAD4. Plant J. 35:193-205.

Fuentes, R. G., Mickelson, H. R., Busch, R. H., Dill-Macky, R., Evans, C. K., Thompson, W. G., Wiersma, J. V., Xie, W., Dong, Y., and Anderson, J. A. 2005. Resource allocation and cultivar stability in breeding for Fusarium head blight resistance in spring wheat. Crop Sci. 45:1965-1972.

Glazebrook, J. 2005. Contrasting mechanism of defense against biotrophic and necrotrophic pathogens. Annu. Rev. Phytopathol. 43:205-227. 
Gorlach, J., Volrath, S., Knauf-Beiter, G., Hengy, G., Beckhove, U., Kogel, K. H., Oostendorp, M., Staub, T., Ward, E., Kessmann, H., and Ryals, J. 1996. Benzothiadiazole, a novel class of inducers of systemic acquired resistance, activates gene expression and disease resistance in wheat. Plant Cell 8:629-643.

Goswami, R. S., and Kistler, H. C. 2004. Heading for disaster: Fusarium graminearum on cereal crops. Mol. Plant Pathol. 5:515-525.

Huang, W. E., Wang, H., Zheng, H., Huang, L., Singer, A. C., Thompson, I., and Whiteley, A. S. 2005. Chromosomally located gene fusions constructed in Acinetobacter sp. ADP1 for the detection of salicylate. Environ. Microbiol. 7:1339-1348.

Huang, W. E., Huang, L., Preston, G. M., Naylor, M., Carr, J. P., Li, Y., Singer, A. C., Whiteley, A. S., and Wang, H. 2006. Quantitative in situ assay of salicylic acid in tobacco leaves using a genetically modified biosensor strain of Acinetobacter sp. ADP1. Plant J. 46:1073-1083.

Kloek, A. P., Verbsky, M. L., Sharma, S. B., Schoelz, J. E., Vogel, J., Klessig, D. F., and Kunkel, B. N. 2001. Resistance to Pseudomonas syringae conferred by an Arabidopsis thaliana coronatine-insensitive (coil) mutation occurs through two distinct mechanisms. Plant J. 26:509-522.

Li, G., and Yen, Y. 2008. Jasmonate and ethylene signaling pathway may mediate Fusarium head blight resistance in wheat. Crop Sci. 48:18881896

Lu, Z. X., Gaudet, D., Puchalski, B., Despins, T., Frick, M., and Laroche, A. 2005. Inducers of resistance reduce common bunt infection in wheat seedlings while differentially regulating defence-gene expression. Physiol. Mol. Plant Pathol. 67:138-148.

Makandar, R., Essig, J. S., Schapaugh, M. A., Trick, H. N., and Shah, J. 2006. Genetically engineered resistance to Fusarium head blight in wheat by expression of Arabidopsis NPR1. Mol. Plant-Microbe Interact. 19:123-129.

Makandar, R., Nalam, V., Chaturvedi, R., Jeannotte, R., Sparks, A. A., and Shah, J. 2010. Involvement of salicylate and jasmonate signaling pathways in Arabidopsis interaction with Fusarium graminearum. Mol. Plant-Microbe Interact. 23:861-870.

McMullen, M., Jones, R., and Gallenberg, D. 1997a. Scab of Wheat and Barley: A Re-emerging Disease of Devastating Impact. American Phytopathological Society, St. Paul, MN, U.S.A

McMullen, M.P., Schatz, B., Stover, R., and Gregoire, T. 1997b. Studies of fungicide, application timing, and application technologies to reduce Fusarium head blight and deoxynivalenol. Cereal Res. Commun. 25:779-780.

Métraux, J.-P., Nawrath, C., and Genoud, T. 2002. Systemic acquired resistance. Euphytica 124:237-243.

Mirocha, C. J., Kolaczkowski, E., Xie, W., Yu, H., and Jelen, H. 1998 Analysis of deoxynivalenol and its derivatives (batch and single kernel) using gas chromatography/mass spectrometry. J. Agric. Food Chem. 46:1414-1418.

Norman-Setterblad, C., Vidal, S., and Palva, E. T. 2000. Interacting signal pathways control defense gene expression in Arabidopsis in response to cell wall-degrading enzymes from Erwinia carotovora. Mol. PlantMicrobe Interact. 13:430-438.

Pieterse, C. M. J., Leon-Reyes, A., Van der Ent, S., and Van Wees, S. C. M. 2009. Networking by small-molecule hormones in plant immunity. Nat. Chem. Biol. 5:308-316.

Pritsch, C., Muehlbauer, G. J., Bushnell, W. R., Somers, D. A., and Vance, C. P. 2000. Fungal development and induction of defense response genes during early infection of Wheat spikes by Fusarium graminearum. Mol. Plant-Microbe Interact. 13:159-169.

Pritsch, C., Vance, C. P., Bushnell, W. R., Somers, D. A., Hohn, T. M., and Muehlbauer, G. J. 2001. Systemic expression of defense response genes in wheat spikes as a response to Fusarium graminearum infection. Physiol. Mol. Plant Pathol. 58:1-12.

Reuber, T. L., Plotnikova, J. M., Dewdney, J., Rogers, E. E., Wood, W., and Ausubel, F. M. 1998. Correlation of defense gene induction defects with powdery mildew susceptibility in Arabidopsis enhanced disease susceptibility mutants. Plant J. 16:473-485.

Savitch, L. V., Subramaniam, R., Allard, G. C., and Singh, J. 2007. The GLK1 'regulon' encodes disease defense related proteins and confers resistance to Fusarium graminearum in Arabidopsis. Biochem. Biophys.
Res. Commun. 359:234-238.

Schmelz, E. A., Engelberth, J., Tumlinson, J. H., Block, A., and Alborn, H. T. 2004. The use of vapor phase extraction in metabolic profiling of phytohormones and other metabolites. Plant J. 39:790-808.

Shah, J., Tsui, F., and Klessig, D.F. 1997. Characterization of a salicylic acid-insensitive mutant (sail) of Arabidopsis thaliana, identified in a selective screen utilizing the SA-inducible expression of the tms 2 gene. Mol. Plant-Microbe Interact. 10:69-78.

Shah, J., Kachroo, P., Nandi, A., and Klessig, D. F. 2001. A recessive mutation in the Arabidopsis SSI2 gene confers SA- and NPRI-independent expression of $P R$ genes and resistance against bacterial and oomycete pathogens. Plant J. 25:563-574.

Skadsen, R. W., and Hohn, T. M. 2004. Use of Fusarium graminearum transformed with gfp to follow infection patterns in barley and Arabidopsis. Physiol. Mol. Plant Pathol. 64:45-53.

Spoel, S. H., Johnson, J. S., and Dong, X. 2007. Regulation of tradeoffs between plant defenses against pathogens with different lifestyles. Proc. Natl. Acad. Sci. U.S.A. 104:18842-18847.

Staswick, P. E., and Tiryaki, I. 2004. The oxylipin signal jasmonic acid is activated by an enzyme that conjugates it to isoleucine in Arabidopsis. Plant Cell 16:2117-2127.

Staswick, P. E., Yuen, G. Y., and Lehman, C. C. 1998. Jasmonate signaling mutants of Arabidopsis are susceptible to the soil fungus Pythium irregulare. Plant J. 15:747-754.

Thomma, B. P. H. J., Eggermont, K., Penninckx, I. A. M. A., Mauch-Mani, B., Vogelsang, R., Cammue, B. P. A., and Broekaert, W. F. 1998. Separate jasmonate-dependent and salicylate-dependent defense-response pathways in Arabidopsis are essential for resistance to distinct microbial pathogens. Proc. Natl. Acad. Sci. U.S.A. 95:15107-15111.

Thomma, B. P. H. J., Eggermont, K., Tierens, K. F. M.-J., and Broekaert, W. F. 1999. Requirement of functional Ethylene-Insensitive 2 gene for efficient resistance of Arabidopsis to infection by Botrytis cinerea. Plant Physiol. 121:1093-1101.

Thomma, B. P. H. J., Eggermont, K., Broekaert, W. F., and Cammue, B. P. A. 2000. Disease development of several fungi on Arabidopsis can be reduced by treatment with methyl jasmonate. Plant Physiol. Biochem. 38:421-427.

Urban, M., Daniels, S., Mott, E., and Hammond-Kosack, K. 2002. Arabidopsis is susceptible to the cereal ear blight fungal pathogens Fusarium graminearum and Fusarium culmorum. Plant J. 32:961-973.

Van Hemelrijck, W., Wouters, P. F. W., Brouwer, M., Windelinckx, A., Goderis, I. J. W. M., De Bolle, M. F. C., Thomma, B. P. H. J., Cammue, B. P. A., and Delauré, S. L. 2006. The Arabidopsis defense response mutant esal as a model to discover novel resistance traits against Fusarium diseases. Plant Sci. 171:585-595.

Verhagen, B. W. M., Glazebrook, J., Zhu, T., Chang, H. -S., van Loon, L. C., and Pieterse, C. M. J. 2004. The transcriptome of rhizobacteriainduced systemic resistance in Arabidopsis. Mol. Plant-Microbe Interact. 17:895-908.

Walter, S., Nicholson, P., and Doohan, F. M. 2010. Action and reaction of host and pathogen during Fusarium head blight disease. New Phytol. 185:54-66.

Wasternack, C. 2007. Jasmonates: An update on biosynthesis, signal transduction and action in plant stress response, growth and development. Ann. Bot. 100:681-697.

$\mathrm{Xu}, \mathrm{X}$., and Nicholson, P. 2009. Community ecology of fungal pathogens causing wheat head blight. Annu. Rev. Phytopathol. 47:83-103.

Yu, G. Y., and Muehlbauer, G. J. 2001. Benzothiadiazole-induced gene expression in wheat spikes does not provide resistance to Fusarium head blight. Physiol. Mol. Plant Pathol. 59:129-136.

Zimmerli, L., Métraux, J.-P., and Mauch-Mani, B. 2001. $\beta$-Aminobutyric acid-induced protection of Arabidopsis against the necrotrophic fungus Botrytis cinerea. Plant Physiol. 126:517-523.

\section{AUTHOR-RECOMMENDED INTERNET RESOURCE}

The GNU Image Manipulation Program (GIMP 2.6) software: www.GIMP.org 